\title{
INICIAR A LOS ALUMNOS EN LA INVESTIGACIÓN Y ELABORACIÓN DE TRABAJOS EN EL CONTEXTO DEL EEES: LA EXPERIENCIA DE LA ASOCIACIÓN FUNDAMENTO JURÍDICO
}

\author{
FRANCISCO DE LA FUENTE CARDONA \\ Graduado en Derecho. Máster en D. Internacional \\ fcfuente@ucm.es \\ DiEGo VigIL DE QUIÑONES OTERO \\ Registrador de la Propiedad. Profesor asociado de D. Civil \\ dvigil@registradores.org
}

Universidad Complutense de Madrid

\begin{abstract}
Resumen
El nuevo Espacio Europeo de Educación Superior (EEES) promueve un cambio en el modelo de adquisición de conocimientos y habilidades, lo cual ha redefinido la metodología docente que tradicionalmente se aplicaba y ha atribuido al alumno un papel protagonista en el denominado "aprendizaje". La experiencia de la asociación Fundamento Jurídico puede constituir un ejemplo de cómo completar las competencias propuestas pero no totalmente adquiridas en el nuevo programa de grado en Derecho, pues tres cursos después de la puesta en marcha de la asociación los resultados son moderadamente positivos.
\end{abstract}

Palabras clave: Fundamento Jurídico, asociación,, docencia, competencia, EEES.

\begin{abstract}
The European Higher Education Area (EHEA) promotes a change in the acquisition of knowledge and abilities which have redefined the traditional teach methodology and the student has acquired a central role in the so called "learning" process. The experience of Fundamento Jurídico association may build a tool to develop the skills that are not always successfully completed in the current law bachelor, due to the fact that after three year the results are moderately positives.
\end{abstract}

Key words: Fundamento Jurídico, association, teaching, competence, EHEA.

\section{Sumario}

1 Introducción: relato breve de una experiencia. 2. El origen de cómo surgió todo. 3. El método de trabajo: estudiar en compañía. 4. Estudiar en amistad. 5. La estética de la experiencia. 6. La experiencia en el contexto del EEES: complemento del nuevo sistema. 7. Experiencia y métodos jurídicos. 8. Conclusiones: un método a imitar. 


\section{Introducción: relato breve de una experiencia.}

La Facultad de Derecho de la Universidad Complutense de Madrid, la misma que antes de crearse las Universidades periféricas llegó a superar en matriculados la población de Teruel, la que es como la casa madre de todas las Facultades de Derecho de España (pues quien más quien menos tiene "padre" o "abuelo" académico con doctorado en la Central), incorporó el plan Bolonia en el otoño de 2010, con alumnos nacidos en 1992. Hasta 2014 rigió, durante 60 años, el plan 53, que tantas satisfacciones ha dejado en tantos ${ }^{1}$. Una Facultad acostumbrada a asignaturas anuales, a ser casa de grandes maestros, a que te diese clase el autor del Manual, una Facultad en la que los mejores alumnos estudiaban, con tiempo para digerirlos, tratados de más de mil páginas por asignatura, incorporaba un plan pensado de otra manera, en el cual más o menos había que meter el viejo plan 1953 en tres cursos, y luego tener un cuarto de optativas (lo cual permite cosas tan atractivas como una cuatrimestral de concursal -dada por Juana Pulgar-, una de daños -dada por Mariano Yzquierdo-, una de hipotecario, una de contratación -con María Teresa Álvarez Moreno-, un mercantil centrado en la PYME, una asignatura separada de urbanismo o una optativa de Derecho penal internacional -a cargo del profesor Ollé Sesé-).

Insertados en dicha tradición de grandes maestros y grandes tratados, los alumnos más capaces del "plan Bolonia" han seguido estudiando los mejores y grandes libros, pero en menos tiempo. Y alcanzado un cierto saber, ha ocurrido que a partir de segundo (cuando ya han estudiado la mitad del antiguo plan) se han visto manejando el Derecho de forma que la realidad les ha pedido que se inicien en la investigación, y comiencen a publicar trabajos.

Consecuencia de ello nació en 2014 la Asociación Fundamento Jurídico², que ha dado como fruto ya tres números de una revista con el mismo nombre, y docenas de

\footnotetext{
${ }^{1}$ Sin pretensión de exhaustividad, son dignos de mencionar los recuerdos sobre la Facultad contenidos en las siguientes obras:

De políticos, FRAGA IRIBARNE, M. Memoria breve de una vida pública. Planeta, Barcelona 1983, que relata algunos aspectos de la Universidad en los años 40. Más amplio (pues más vida tenía en aquellos momentos la Facultad) es el relato que se hace de la vida de la Facultad en los años 50 y primeros 60 por el Letrado del Consejo de Estado y ex Ministro, que fue alumno y profesor, ORTEGA DIAZ-AMBRONA, J.A Memorial de transiciones, Galaxia Gutemberg, Madrid 2015 (especialmente Págs. 115 a 123 y 131-132).

Centradas en recuerdos de los maestros, GARRIGUES DÍAZ- CAÑABATE, J. "Don Felipe y la Universidad". Revista de Derecho Privado, t. XXIX, 1945, p. 618.

PEÑA BERNALDO DE QUIRÓS, M "En memoria de Don Federico de Castro y Bravo, nuestro Director". Anuario de Derecho Civil. XXXVI-II. 1983, abril-junio, pags 353 y ss. Este texto aparece recogido en Derecho Civil: estudios, comentarios y notas. Tomo I. Colegio de Registradores 2009. pags. 67 y ss.

RICO PÉREZ, F. "Los miércoles jurídicos de Don Federico de Castro". En Estudios Jurídicos en Homenaje al Profesor Diez- Picazo. Civitas. Madrid 2003.

MORO SERRANO, J.L. "En recuerdo de mi compañero y amigo Manuel Amorós Guardiola" En revista Registradores de España, número 53, Noviembre- Diciembre 2009, páginas 36 y 37;; así como el relato de Manuel PEÑA en

Aun cuando se centra más en el ambiente de las oposiciones, es digno de destacar el relato entorno a la figura de don Eduardo GARCÍA DE ENTERRÍA contenido en FERNANDEZ- CARVAJAL, P. "Retrato de Eduardo joven". En Estudios sobre la Constitución española: homenaje al Profesor Eduardo García de Enterría. Civitas, 1991. Pag. XXX.

${ }^{2} \mathrm{http}: / /$ www.asociacionfundamentojuridico.com/
} 
pequeños post sobre temas de más inmediata actualidad ${ }^{3}$. Y así, al igual que en una escuela de arquitectura es normal encontrar maquetas elaboradas por los alumnos, se produce algo que resulta incompresible que no haya llegado antes a las Facultades de Derecho: una exposición pública de los primeros escritos de alumnos.

Además de contenidos de investigación, la indicada Asociación ha entrevistado entre otros a Antonio Garrigues Walker, o Carlos Lesmes; ha organizado diversas visitas a instituciones; y ha organizado una conferencia sobre la protección del consumidor del Director General de los Registros y el Notariado, Francisco Javier Gómez Gálligo.

Es en esa tradición, en esa Facultad (tradicionalmente acostumbrada a asociaciones políticas ${ }^{4}$ o de otro tipo, pero no de alumnos con inclinación a la investigación), en ese ambiente de compañeros, es donde ha surgido dicha asociación. Un fenómeno del que vale la pena dar cuenta, aunque sus protagonistas sean tan jóvenes, pues tal vez la promoción de asociaciones similares pudiese ser útil a otros centros para el mismo fin de acoger y potenciar dicha inclinación investigadora y para promover el "aprendizaje" del alumno en el nuevo marco del EEES, pues éste exige a los docentes no sólo enseñar conceptos e instituciones jurídicas sino "enseñar a aprender", mientras que posiciona a los alumnos con un papel proactivo en su formación que ya no sólo va a consistir en estudiar el Manual del profesor.

\section{El origen de cómo surgió todo.}

La Facultad de Derecho de la Universidad Complutense ha sido, es y seguirá siendo lo que hoy algunos denominan un think tank aunque sus paredes e instalaciones (recientemente en proceso de mejora) se levantasen en una época donde la lengua de W. Shakespeare no inundaba nuestro día a día como requirement para casi todo.

Durante el curso 2013-2014 en la Facultad, fruto de años de apuesta por ello, adquiere todavía un mayor peso y visibilidad la Sociedad de Debate, que tantas alegrías y orgullos nos ha dado en los últimos años. En ese contexto de actividad por parte de los estudiantes (con el constante apoyo de las autoridades de la Facultad) ${ }^{5}$, de sentir que hay que aprovechar la oportunidad de estar en esa casa, en un ambiente de sinergias innovadoras y constructivas, es en el que surge la idea de iniciar a los alumnos de grado en la investigación como una actividad complementaria a las asignaturas impartidas en el programa oficial adaptado al Espacio Europeo de Educación Superior (EEES), al que nos referiremos más adelante.

\footnotetext{
${ }^{3}$ https://www.facebook.com/ucmfj/

${ }^{4}$ Resulta interesante el capítulo que dedicado a los jóvenes liberales de los 80 en la Facultad (agrupados en la Asociación 1812), que luego fueron clave en el PP liderado por Aznar, por DE DIEGO VILLAGRÁN, E. en Pretorianos, de donde vienen y a donde van los fontaneros de la Moncloa (Martinez Roca, Madrid 2004). Según reveló a uno de los coautores de este artículo Gabriel Elorriaga Pisarik (uno de aquellos jóvenes liberales), en un acto reciente en la Real Academia, es el único libro del que tiene noticia que habla de este tiempo de los liberales ochentenos en la Facultad.

${ }^{5} \mathrm{Si}$ para el futuro desde la Facultad de Derecho de la UCM se plantease elaborar un proyecto educativo de centro como por ejemplo menciona la Universidad Carlos III, sin duda, el asociacionismo debería ser uno de los elementos que configuraría dicho proyecto. Sobre la idea de proyecto de centro educativo véase TURULL RUBINAT, M, ALBERTÍ ROVIRA, E, 'El 'proyecto educativo de centro' en el ámbito universitario. Reflexiones y propuestas a partir de la experiencia de la Facultad de Derecho de la Universidad de Barcelona (2005-2016), Revista de Educación y Derecho, nº15, 2016.
} 
Se trató de un proceso que los estudiantes vivieron con mucha ilusión desde un primer momento. Las relaciones como compañeros habían sentado ciertas bases durante el primer curso de grado, en ese momento en que el uno aterriza en la Universidad con dos frentes abiertos, (i) a nivel personal, pues con dieciocho años toda persona se encuentra en pleno desarrollo de su personalidad y le preocupa encontrar personalidades afines, y (ii) a nivel académico, pues el sistema de Bachillerato cambia en profundidad, aunque hemos de reconocer que desde la introducción del denominado plan Bolonia, empieza a dejar de ser una rara avis que los Profesores conozcan a sus alumnos por nombre y apellidos, algo difícilmente imaginable en aquellas aulas de cientos de alumnos.

El segundo curso se presentaba, por tanto, como el momento ideal para iniciar un proyecto de estas características, y así fue. Daniel Olivares, Guillermo Blanco y Francisco de la Fuente nos planteamos cómo presentar la idea con cierto atractivo para que otros compañeros, de los que éramos conocedores de su calidad personal y de su seriedad como estudiantes, se sumasen a este proyecto que por el momento solo residía en nuestras cabezas. En diciembre de 2013 la Asociación Fundamento Jurídico quedó inscrita en el Registro de Asociaciones de la Comunidad de Madrid y entre el otoño e invierno de ese curso se incorporaron Víctor Corpa, Gabriel Fernández, Palmira Galilea y Pablo Méndez-Monasterio (por orden alfabético). Desde aquel momento y hasta el día de hoy quedaron identificadas tres grandes áreas de trabajo, pues el devenir de los anteriores cuatrimestres había demostrado que eran difícilmente realizables en el seno de cualquier asignatura donde los límites temporales apremiaban desde el primer día. Estas tres grandes áreas fueron, en primer lugar, la redacción de artículos de investigación y de noticias jurídicas de actualidad (bien individual bien colectivamente); en segundo lugar, la realización de entrevistas a juristas de reconocido prestigio; y por último, organización de visitas y contacto con instituciones así como un compromiso y colaboración con la Universidad Complutense por habernos brindado tal oportunidad. El resultado de estas áreas de trabajo se recoge en la revista (homónima a la asociación) publicada en versión papel y electrónica, disponible al público en la página web.

Paralelamente, durante esos meses contactamos con Profesores con los que habíamos establecido buena relación durante el primer curso o de asignaturas que estábamos cursando ${ }^{6}$. Creemos que no exageramos si afirmamos que desde el primer momento se obtuvo de forma unánime su apoyo y visto bueno al proyecto Fundamento Jurídico. Y así, la asociación comenzó a andar con el primer acto de presentación de los artículos de investigación el lluvioso jueves 24 de abril de 2014, agradeciendo a todos los compañeros de curso y Profesores su asistencia.

\section{El método de trabajo: estudiar en compañía.}

Conformado el grupo inicial, se comenzó a cooperar compartiendo lectura, comentando las ideas que se iban generando en las mismas, elaborando los primeros escritos, que gustosamente se leían unos a otros.

\footnotetext{
${ }^{6}$ Lo que significó la posibilidad de superar, aun parcialmente, lo que refleja BOTO ÁLVAREZ, A, en "El potencial de las revistas jurídicas de estudiantes en el EEES", Docencia y Derecho, nº 4, 2012. Pág. 2 pues indica que las revistas estudiantiles "se enfrentan a serias dificultades por la escasez de medios y fondos, de manera que su edición implica un fuerte compromiso y por ello el resultado final suele acabar rayando en lo reivindicativo y outsider, de forma que suele pasar desapercibido para los docentes y se ignora más allá de los pasillos".
} 
La Universidad muchas veces vive en la insolidaridad, en la masificación, en la falta de trato. Algo que no es sólo culpa de los alumnos, sino también de la estructura. Algunas Universidades del pasado (de las que nos han llegado relatos más idealizados de la cuenta) no padecían esto porque eran pequeñas y residenciales. Todos se conocían entre los diferentes Colegios Mayores y la relación entre profesores y alumnos a través del llamado "sistema tutorial" brotaba sola al unirse todos en un espacio.

Resulta curioso que el Código de las Siete Partidas regulaba específicamente en la Partida II, entre las formas de agrupación, villas, señoríos, cargos y ámbitos de poder, las Universidades, definiéndolas como "ayuntamiento de maestros y escolares con voluntad de entendimiento de aprender los saberes"7.

Reflexionando sobre si la Universidad debía ser residencial- tutorial, o no, NEWMAN comparaba la Universidad de Londres de su tiempo, de la que decía como algo cuestionable que "otorga sus títulos a cualquier persona que haya aprobado un examen" y la contraponía a la Universidad de Oxford, de la que se llegó a decir en siglo XVIII que "juntaba unos cuántos jóvenes tres o cuatro años y luego los despedía", modelo que (si hubiese que elegir) le era preferido ${ }^{8}$.

La Universidad residencial es una gran idea a la que no tenemos por qué renunciar mientras queden Colegios Mayores. Ahora bien, formadas las grandes urbes, expandida la Universidad y convertida en servicio accesible en el Estado del bienestar, la residencia no puede con todos los alumnos ${ }^{9}$. ¿Qué hacer con los demás? Fomentar grupos pequeños y clubes al estilo de las Universidades americanas para alumnos de la ciudad no residentes en la Universidad sería una solución ${ }^{10}$.

Si además dichas asociaciones, en lugar de gestionar los primeros pasos políticos de los alumnos, o su participación cultural o deportiva, son el cauce de una inclinación a la investigación, entonces cooperan mucho mejor al fin de la Universidad que la simple estructura burocrática o mercantil hoy existente.

Fundamento Jurídico ha sido esto.

\section{Estudiar en amistad.}

Ello nos debe llevar a una mínima profundización sobre el papel de la amistad en los estudios de Derecho.

Como ha sostenido uno de nosotros en otro lugar ${ }^{11}$, un elemento comprobado del éxito en los estudios jurídicos es la colaboración de varias personas. El Derecho es muy complicado, son muchos los matices y siempre pueden ser varias las interpretaciones. En tales condiciones, es casi imposible que un solo ser humano sea capaz de conocerlo todo y dar solución a todo. El estudio solitario es bueno y necesario, pero la complejidad del Derecho, nos coloca a todos en una posición limitada. Un límite

\footnotetext{
${ }^{7}$ Partida II, título XXI.

${ }^{8}$ Cfr. KER, I. Idea de una Universidad en Newman. Traducción de Javier Martín Valbuena, que se remite a la obra The Idea of University. Pag. 10

${ }^{9}$ Según datos recientes, sólo el $1 \%$ de los universitarios reside.

${ }^{10}$ Una solución que requeriría miles de iniciativas. Piénsese que pese a su carácter minoritario, los Colegio Mayores de Madrid organizaron más de 650 iniciativas el pasado curso. Lograr tener "actividad extracurricular" requeriría un esfuerzo a gran escala.

${ }^{11}$ VIGIL DE QUIÑONES OTERO, D. Breves consejos para estudiar Derecho con éxito. $2^{\mathrm{a}}$ Ed. Visión Libros. Madrid 2014. Págs. 29 a 34. Para una aplicación concreta al estudio en las oposiciones, ver VIGIL DE QUIÑONES OTERO, D. Consejos para opositar con éxito. CEU Ediciones, Madrid 2011, Págs. 56 a 61.
} 
que se supera con la colaboración de varias personas. Al igual que en la realización del Derecho se requiere la intervención de diversos agentes que representen a las posturas en conflicto para dar una solución lo más justa posible (así los Abogados de las partes y el Juez; el Fiscal; el Notario, el Registrador; los órganos consultivos etc), la mutua cooperación de compañeros de estudios ${ }^{12}$, permite compartir dudas, aclararlas, beneficiarse unos de los descubrimientos y logros de los otros. Además, en ésas conversaciones se van forjando ilusiones, se intercambia información,....y con ello se va trazando el futuro de cada uno viviendo una grata amistad ${ }^{13}$.

Pero todo empieza por un encuentro de cada persona con las demás. Un encuentro en el que se descubre que hay otras personas con intereses y vocación comunes. La apertura a la conversación y a la colaboración con otras personas propia de una asociación, además de proporcionar disfrute, coloca en posición de estudiar Derecho mejor, pues la dificultad de la materia se suple con la colaboración de varios (que siendo amigos, colaborarán pues quieren el bien de los demás ${ }^{14}$ ).

Casi se podría decir que hay para estudiar bien Derecho, se debe estudiar en amistad, sobre todo si valoramos esta, no como querer lo mismo, si no en obrar de modo conjunto, y como un ámbito de relación en lo que importa, no sólo los intereses, si no la grandeza $^{15}$ y la belleza de la vida ${ }^{16}$. Y para ello, la experiencia asociativa ha resultado capital.

\section{La estética de la experiencia.}

Un aspecto interesante que no querríamos dejar de mencionar es el de la estética. La revista Fundamento Jurídico se ha procurado editar con el mayor de los cuidados. Los actos de la Asociación se ha pretendido que sean siempre en salas nobles de la Facultad, y los alumnos asociados se han auto-impuesto la obligación de acudir con traje y corbata ellos, y con vestido o traje ellas.

¿Por qué?, ¿para qué? Los alumnos asociados siempre quisieron expresar el clima de aprecio y confianza que reinó entre ellos, el anhelo de excelencia, y la belleza de lo que estaban haciendo.

Con carácter general, se entiende que la belleza es la expresión del bien susceptible de producir agrado. Desde dicha concepción, todas las instituciones jurídicas, en tanto esenciales para el bien que constituye la correcta administración de la Justicia y la mejor organización de la comunidad, han establecido una estética determinada. En todos los países y en todas las civilizaciones, los palacios de Justicia han sido lugares centrales y relevantes, se han observado formalidades cuyo único papel

\footnotetext{
${ }^{12}$ Indica C. S. LEWIS (autor de "Las crónicas de Narnia") que el compañerismo es "la matriz de la amistad", pues esta surge "cuándo dos o más compañeros descubren que tienen en común algunas ideas o intereses o simplemente algunos gustos que los demás no comparten y que hasta ese momento cada uno pensaba que era su propio y único tesoro, o su cruz" (LEWIS, C.S Los cuatro amores. $12^{\circ} \mathrm{ed}$. Rialp. Madrid 2008. Págs. 77 y 78).

${ }^{13}$ Vale la pena leer los testimonios de amistad de las obras que hemos citado al comienzo, referidas a la historia de la Facultad de Derecho de Madrid.

${ }^{14}$ Dice ARISTÓTELES que "la amistad perfecta es la de los hombres buenos a iguales en virtud; porque estos quieren el bien el uno del otro en cuanto son buenos y son buenos en sí mismos; y los que quieren el bien de sus amigos por causa de éstos, son los mejores amigos" Cfr. Ética a Nicómaco. IX, 6.

15 "Porque es ahí donde el corazón puede abrirse a la magnanimidad" (NORIEGA BASTOS, J. "La concordia o la metamorfósis del corazón". En En GRANADOS GARCÍA, C. y GRANADOS GARCÍA, J. El Corazón: urdimbre y trama. Colección Didaskalos. Ed. Monte Carmelo. Burgos 2010. Pag. 125).

${ }^{16}$ En el sentido que lo explica ARISTÓTELES, Ética a Nicómaco. IX, 3.
} 
era solemnizar la administración de la Justicia y se han fijado formas determinadas (normalmente vistosas, agradables y con pretensión de ser "bellas") para Jueces y Abogados. Estas cualidades no sólo afectan al ámbito judicial, sino también al extrajudicial. En efecto, toda institución que desempeña un papel en la realización de la Justicia (despachos de Abogados, Notarías, Administraciones, Registros jurídicos), suelen presentar una estética apropiada para transmitir al usuario el valor y la importancia de lo que se está haciendo o recibiendo, del servicio que se presta, del resultado que se tiende a conseguir ${ }^{17}$.

A la luz de lo anterior, debemos valorar la situación de la estética en las Facultades de Derecho. Pese a lo claro que resulta la conexión belleza-justicia en la forma estética de las instituciones, sin embargo son pocas las Facultades de Derecho en las que la estética sea la apropiada a lo que es luego la de las instituciones en las que van a desempeñar un valioso papel los estudiantes que salgan de las mismas.

Una forma de mejorar la vida de las Facultades, podría ser mejorar su estética: tanto la de los edificios ${ }^{18}$ como la de las personas en su forma de vestir, de tratar, de comportarse... ${ }^{19}$.

Esta mejora contribuirá al éxito individual y colectivo de cada alumno y de cada uno de los centros. Frente a ello se podrá objetar que da lo mismo cuidar la estética que no cuidarla, que se puede cuidar llegado el momento de incorporarse al mercado de trabajo, o que no es preciso aplicar dicha exigencia a la vida de los estudiantes. Sin embargo, lo cierto es que los centros educativos que cosechan mejores resultados en el mundo (y las Facultades de Derecho no son una excepción) son siempre los que mejor cuidan la estética.

Aunque parezca mentira, detrás de la estética hay otros elementos que, aunque no se suele tomar conciencia de ello, están relacionados con la misma. En efecto, si un alumno cree que no le importa a nadie, no viste igual que si sabe que el entorno, los compañeros, las personas...le aprecian. La forma de vestir personal y la decoración de los habitáculos, son importantes para todo ser humano. A todo el mundo le gusta decorar de algún modo que le resulte agradable su despacho o su habitación. Del mismo modo, no se elige el mismo lugar ni la misma ropa para actividades cotidianas que para actividades solemnes: la forma de vestir y el lugar son siempre mejores para lo que más se valora (por ejemplo, celebraciones tales como bodas o actos de graduación). Si esto es así, en la enseñanza también tiene aplicación: la forma de vestir es exteriorización del aprecio que le dan las personas al grupo con el que trabajan y a la actividad que desarrollan. Si se cuidan el compañerismo y la amistad en la línea indicada, lo normal será que surja cierta tendencia a vestir de modo más cuidado. En tanto ese aprecio va ligado en este caso a una inclinación profesional, se vestirá cada vez más acorde a los usos de ese mundo profesional al que se preparan los estudiantes de Derecho. Ello llevará a cuidar más el entorno de las Facultades. Y

\footnotetext{
17 Veánse las páginas dedicadas a la estética de la Justicia en LÓPEZ QUINTÁS, A. Estética de la creatividad. Juego. Arte. Literatura. Cátedra, Madrid 1977. Pág. 285.

${ }^{18}$ El nuevo equipo decanal de Derecho de la UCM ha decidió recientemente adornar el hall con un gran piano de cola sobre una moqueta granate- Puede verse la noticia en ABC:

http://www.abc.es/espana/madrid/abci-tocar-steinway-hall-derecho-201612130032_noticia.html

${ }^{19}$ Sobre éste punto es interesante consultar el trabajo ROCA, M.J. "La Forma estética personal y la cultura humanística universitaria”. Foro Nueva Época 4/2006, 211-220. También publicado en Diálogo filosófico, 68 (2007), pp. 269- 278.
} 
esa mejoría, normalmente, redundará en beneficio de la carrera de quien contribuye a elevar el nivel humano de su clase mediante el cuidado de su de su vestir o de su trato.

Aunque lo que estamos diciendo puede parecer el camino inverso, no es rechazable afrontarlo. En efecto, lo normal es que la estética brote del interior y no que a base de cuidar la estética se vaya a mejorar el interior. Pero, como indica la

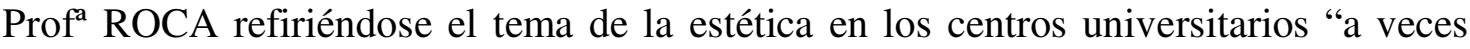
tratar los síntomas contribuye notablemente a mejorar el estado del enfermo hasta el punto de que se encuentre mejor para acometer su propia curación"20.

En efecto, las Facultades de Derecho mejorarían en la medida en que nos propusiésemos cuidar su estética. Y es que como recuerda dicha autora, citando a Von BALTHASAR, no hay formación humana fecunda que pueda prescindir de lo bello ${ }^{21}$.

Ahora bien, ¿cómo lograr éste cuidado? Para ello, siguiendo el planteamiento de la autora citada, harían falta dos cosas. Lo primero es crear un clima de confianza: quien valora a los demás, procura resultarles agradable (también en su trato y en su forma de vestir). En segundo lugar, es necesaria una estima por la institución en la que se estudia: quien no valora como propia la (su) Universidad, difícilmente va a cuidarla. $\mathrm{Y}$ es que como dice el magnate de la moda de lujo española Enrique LOEWE "el mayor freno y la mayor sanción que tiene la moda es que te de igual, la indiferencia" 22 . Lo que decimos no tiene que ver tanto con la marca cuanto con el estilo, pues no se puede ignorar que hoy por hoy las grandes multinacionales (como Inditex) ofrecen líneas que se ajustan a esa concepción de cuidado situándolas al alcance de amplios segmentos de la sociedad ${ }^{23}$, por lo que esta idea de estética respetaría la igualdad de oportunidades de que puede presumir la Universidad Pública en la medida en que podría participar de ella todo estudiante que así lo desee.

Considerados estos aspectos, podríamos decir que la estética vivida por los jóvenes miembros de Fundamento Jurídico ha sido clara expresión de su aprecio por lo que hacían, por si mismos, de la confianza entre ellos, y de su determinación clara de

${ }^{20}$ Ob. Cit. Pag. 212.

${ }^{21}$ BALTHASAR. H. von, Herrlichkeit. I. Schau der Gestalt, 1961, trad. española, Gloria, vol. 1. La percepción de la forma, Madrid, 1985, p. 16.

22 Mesa Redonda Moda, personalidad, valores. Bilbao, 21 de Marzo de 2009. En dicha intervención, el Presidente de la Fundación Loewe lanzó otras dos ideas que nos parecen dignas de consideración. La primera es que "la elegancia también se aprende", lo cual es muy importante para todo alumno, en especial si es alguien procedente de un ámbito social donde no se trabaja la estética igual que en la minoría de los juristas: el alumno debe saber que igual que es capaz de penetrar en los conocimientos del Derecho, puede penetrar en el conocimiento de la estética propia de las instituciones jurídicas.

La segunda idea es que no podemos ser mediocres y alienados, sino que tenemos que aspirar a un lujo, a inventarse y vivir como personas, y a descubrir lo que a uno realmente le gusta. Esta idea es importante por cuánto los tiempos de crisis o la decadencia en el aprecio de las instituciones pueden llevar a un empobrecimiento estético de las mismas. Contrario modo, mantener el cuidado y la creatividad pueden ser determinantes del mantenimiento del aprecio en el futuro. En éste orden debemos destacar como muy positivo el esfuerzo llevado a cabo por muchas instituciones como determinados Colegios Profesionales y Administraciones que han ido renovando su decoración, su estética, manteniendo la excelencia en nuevas formas y sabiendo invertir en cuidar y restaurar elementos antiguos que contribuyen a crear una imagen agradable (bella) a la que asirse para valorar el bien de determinadas instituciones en tiempos en los que parece que todo se cuestiona.

${ }^{23}$ En lo que cierta doctrina no ha dudado en calificar de "mayoría selecta". Cfr. GOMA LANZON, J. "Mayoría selecta" en El Pais, 12 de Enero de 2013. 
insertarse en el mundo del Derecho (a base de imitar su estética habitual) y de seguir su inclinación a la investigación (cuidando también las mejores expresiones de la estética universitaria).

\section{La experiencia en el contexto del EEES: complemento del nuevo sistema.}

La sustitución del plan del 53 por los programas elaborados como consecuencia del denominado plan Bolonia o Espacio Europeo de Educación Superior (EEES) es el resultado de una visión de los estudios de grado como determinantes de la adquisición de las necesarias competencias ${ }^{24}$ para la integración de los estudiantes en el mercado de trabajo apostando por la idea de "aprendizaje" en la que el alumno tiene un papel activo y no de mero receptor (lo cual exige nuevas metodologías docentes) ${ }^{25} \mathrm{y}$, por otro lado, de la intención de que los sistemas de los diferentes Estados de la Unión Europea converjan para impulsar la materialización de los principios formales antes comunitarios y hoy recogidos en el TUE y en el TFUE ${ }^{26}$.

La primera de estas cuestiones, esto es, la referida al papel del alumno y a la adquisición de competencias y habilidades que refuercen los objetivos propuestos por el actual grado es donde Fundamento Jurídico puede servir como experiencia. En la memoria verificada del grado en Derecho de la Universidad Complutense ${ }^{27}$ se recogen una serie de competencias básicas entre las que destacamos "que los estudiantes tengan la capacidad de reunir e interpretar datos relevantes (normalmente dentro de su área de estudio) para emitir juicios que incluyan una reflexión sobre temas relevantes de índole social, científica o ética" y "que los estudiantes hayan desarrollado aquellas habilidades de aprendizaje necesarias para emprender estudios posteriores con un alto grado de autonomía", es decir, el alumno no se va a limitar a acudir a clase y estudiar el manual correspondiente, sino que debe ir un paso más allá e iniciarse en el uso de bases de datos jurisprudenciales o doctrinales, en la resolución de casos prácticos (pues en este sistema de educación donde la unidad de medida es el ECTS -European Credit Transfer Systeméstas tienen asignado un papel vertebral en la evaluación) o la redacción de informes sobre cuestiones al alcance de su etapa formativa ${ }^{28}$.

\footnotetext{
${ }^{24}$ En el presente trabajo no abordaremos el debate sobre el concepto y clasificación de las competencias, pues es merecedor de un lugar autónomo.

25 "No se espera que se desempeñe el mismo trabajo toda la vida ni que las demandas de los diferentes trabajos sean idénticas. Por tanto, es necesario preparar a los estudiantes para que puedan adaptarse a diferentes condiciones laborares situadas todas ellos dentro del marco de las cualificaciones o niveles formativos superiores. Esto supone que los estudiantes deben aprender y ser entrenados en el manejo de competencias" PAREJO GUZMÁN, M.J, "Innovaciones docentes mediante competencias en las ciencias jurídicas en el espacio europeo de educación superior”, Docencia y Derecho, n², 2010. Pág. 20.

${ }^{26}$ Impregnada por esa finalidad en pro de la construcción europea comienza la "Declaración conjunta de los ministros europeos de educación reunidos en Bolonia el 19 de junio de 1999", disponible en http://www.eees.es/pdf/Bolonia_ES.pdf ; Hoy, el artículo 165.1 primer apartado TFUE reza que "la Unión contribuirá al desarrollo de una educación de calidad fomentando la cooperación entre los Estados miembros y, si fuere necesario, apoyando y completando la acción de éstos en el pleno respeto de sus responsabilidades en cuanto a los contenidos de la enseñanza y a la organización del sistema educativo, así como de su diversidad cultural y lingüística".

${ }^{27}$ Disponible en https://derecho.ucm.es/data/cont/media/www/pag80830/Memoria\%20Grado\%20Derecho.pdf

28 Resumiendo los resultados tomados de PROSSER y TRIGWELL (2006), GONZÁLEZ, C, MONTENEGRO, H, LÓPEZ, I.M y COLLAO, P, "Relación entre la experiencia de aprendizaje de estudiantes universitarios y la docencia de sus profesores”, Calidad en la Educación, n³35, 2011. Pág. 24 recogen como hallazgo previo a su estudio que "los estudiantes que abordan el aprendizaje de manera
} 
Lo cierto es que estos deseos requieren una carga de trabajo mayor y una implicación con la materia que no siempre se percibe los primeros años de grado. Por ello, hay que desarrollar herramientas que despierten y potencien el interés ${ }^{29}$ por el estudio (en un sentido amplio) y es aquí donde Fundamento Jurídico puede suponer tres aportaciones relativas al papel de los Profesores, a la adquisición y consolidación de competencias y a la contextualización del estudio a través de las actividades extraacadémicas y del contacto con las instituciones.

\section{(i) El papel de los profesores.}

Los profesores son el pilar sobre el descansa el mayor peso dentro de la Universidad. Desde el punto de vista de su papel con respecto al alumno, podemos decir "que [con el EEES] cambia la metodología docente y el modo de orientar la enseñanza. Supuestamente, debe plantearse la docencia dirigiendo la mirada al alumno y su aprendizaje, valorando de modo numérico el trabajo que este ha de desarrollar. Se trata de un modelo de enseñanza centrado más en el aprendizaje (volumen total de trabajo del alumno) que en la docencia (horas de clase), que requerirá el uso de nuevas metodologías docentes más enfocadas al desarrollo de destrezas, habilidades y competencias en el alumno"30. Es decir, con la metodología ABP el docente es tutor del aprendizaje autónomo del estudiante y es éste último quien asume la tarea de generar el conocimiento que va a ser objeto de su propio aprendizaje ${ }^{31}$. Así, se potencian los proyectos de innovación docente en el que uno de nosotros ha podido participar con motivo de la elaboración del Trabajo de Fin de Grado ${ }^{32}$.

Sin embargo, este vocación de introducir nuevas formas de docencia propugnada por el nuevo sistema no creemos que tenga por objetivo abandonar aquellos elementos que se han considerado de forma positiva inherentes a la labor del profesor universitario $^{33}$ como la explicación de las instituciones y de los conceptos jurídicos más importantes (en caso contrario, para ello está la universidad a distancia) o como maestro

profunda tienden a presentar concepciones sofisticadas y percepciones positivas sobre el contexto. Además, son los que tienen mayor probabilidad de obtener mejores resultados académicos".

${ }^{29}$ Nos parece interesante la metodología propuesta por ALASTUEY DOBÓN, C, ESCUCHURI AISA, E, y MAYO CALDERÓN, B, en "Formación en competencias a través del estudio de la jurisprudencia. La experiencia en el Derecho Penal", Docencia y Derecho, n³, 2011, pág. 5 quienes recuerdan que "a lo largo de este proceso [transmisión de conocimientos teóricos para aplicarlos luego al caso práctico] el alumno se va desmotivando, pues pierde de vista la finalidad última de este bagaje teórico".

${ }^{30}$ VIDAL PRADO, C, "El espacio europeo de educación superior y su implantación en las universidades españolas", en Revista catalana de Dret Public, nº44, 2012. Pág. 261.

31 FONT RIBAS, A, LLUÍS CABALLOL, A, GUAL DALMAU, M, GUASCH MARTORELL, R, GÓMEZ TRINIDAD, S, MORALES BARCELÓ, J, PONT VILADOMIU, C, ENZLER FANDÓS, A, "Las dificultades del aprendizaje autónomo en un contexto de ABP", Revista de Educación y Derecho, nº, 2009. Pág. 7.

${ }^{32}$ Sobre las iníciales cuestiones que se plantean en torno a los Trabajos de Fin de Grado en la Facultad de Derecho de la UCM véase GARROTE DE MARCOS, M, "El TFG: sus modalidades, objetivos y competencias a validar. Reflexiones a partir de la experiencia de la Facultad de Derecho de la UCM", Docencia y Derecho, $\mathrm{n}^{\circ} 9,2015$.

33 CUBERO TRUYO, A, "Dificultades para la innovación docente en las áreas jurídicas. La compatibilidad entre la adquisición de competencias profesionales específicas y el cumplimiento de los objetivos globales", Revista de Educación y Derecho, nº, 2009. Pág. 9 advierte que "la principal preocupación del profesor va a tener que consistir en cómo conjugar en las coordenadas temporales de las que dispone la exposición teórica suficiente y la aplicación práctica necesaria”. 
que pueda tener una labor de acompañamiento en el crecimiento personal y académico del alumno.

$\mathrm{Y}$ es en este aspecto donde Fundamento Jurídico ha podido aportar su primera contribución. Como hemos explicado más arriba, creado el primer equipo de alumnos, se buscaron algunos Profesores que sirviesen de apoyo, obteniéndolo generosamente de la entonces vicedecana de alumnos Consuelo Martínez-Sicluna y Sepúlveda, las penalistas Margarita Valle Mariscal de Gante, Margarita Martínez Escamilla, la civilista $\mathrm{M}^{\mathrm{a}}$ Patricia Represa Polo, el coautor de este trabajo, y otros.

Dicha forma de relación con los profesores resultó sumamente novedosa. No se trataba ya de una relación circunscrita a una asignatura, si no que más bien se parecía a la que suele surgir entre maestros y discípulos en el ámbito del doctorado...pero con alumnos de Grado. Se regía más por la auctóritas ${ }^{34}$ que por la potestas (en tanto "saber públicamente reconocido" que diría el romanista $\mathrm{D}^{\prime} \mathrm{ORS}^{35}$, superaba la masificación propia de las entidades públicas, propiciaba más el método socrático (sobre todo en su verdadera vertiente de trato individual ${ }^{36}$ ), y propició por tanto un pleno desarrollo del papel $^{37}$ de los maestros en el acontecimiento educativo ${ }^{38}$.

De modo que podríamos afirmar que una asociación de alumnos con fines precientíficos es además sumamente útil para el Profesorado, quien al prestarle la atención que merece, alcanza un nivel de plenitud en su labor que la propia Universidad es a día de hoy incapaz de proporcionar si nos quedamos sólo en la estructura institucional ${ }^{39}$.

\footnotetext{
${ }^{34}$ Sobre el concepto romano de auctóritas, vid. DOMINGO OSLÉ, R. Auctoritas. Ariel, 1999. El autor escribió un artículo en prensa de ese mismo título “Auctoritas” en El País del 71 de Enero de 1999, descargable en: http://elpais.com/diario/1999/01/07/opinion/915663603_850215.html

${ }^{35}$ D'ORS, A. Derecho privado romano, 8. Pamplona 1997.

${ }^{36}$ SÓCRATES afirmaba que en cuánto maestro él no transmitía la sabiduría pero jugaba en su generación un papel similar al de la partera en el parto. Cfr. PLATON. Diálogos. Teeteto, 149.

37 Señalaba a éste respecto Luigi GUISANNI como algo extraño que se considerase como ideal aquel centro educativo en que el contenido transmitido era más importante que el profesor que transmitía, "pues con ello se le quita a la relación profesor- alumno lo más característicamente humano que hay en ella: la aportación propiamente humana, la genialidad del maestro" (Educar es un riesgo, Ed. Encuentro, Madrid 2006, p.78).

${ }^{38}$ Como indica DE RIBERA “cuando una persona se encuentra con la sabiduría y la reconoce como tal, ésta enlaza de modo interno y profundo con -sus- deseos y afectos, que de alguna manera se sienten partícipes de esa sabiduría y se configuran a base de ella. De este modo, la sabiduría, que es trascendente es engendrada de modo inmanente en el interior de la persona. En ese momento, en que se ha dado la interiorización de la sabiduría, podemos decir que ha tenido lugar plenamente el acontecimiento educativo".DE RIBERA. "Ayudar a engendrar: la figura del maestro en la Paideia Griega". En GRANADOS GARCÍA, J. y GRANADOS GARCÍA, J.A. La alianza educativa. Introducción al arte de vivir. Colección Didaskalos. Ed. Monte Carmelo. Burgos 2009. Pag. 65-66.

${ }^{39}$ Refiriéndose a las nuevas exigencias del EEES en relación al papel del profesorado, por todos RIESCO GONZÁLEZ, M, "El enfoque por competencias en el EEES y sus implicaciones en la enseñanza y el aprendizaje", Tendencias Pedagógicas 13, 2008. Pág. 102 establece que "en esta nueva filosofía, el profesor debe ser...un maestro que guía al alumno en su proceso de desarrollo y de transformación de los datos y la información al conocimiento, con miras a la empleabilidad. Debe centrarse no tanto en la enseñanza cuanto en el aprendizaje autónomo del alumno, proporcionándole ocasiones, estrategias y herramientas adecuadas"; IBARRA SERRANO, F.J, "La formación jurídica en el S.XXI", en IBARRA SERRANO, F.J, ROJAS CASTRO, M.O, PINADO SOLORIO, M.E, La educación jurídica. Retos para el siglo XXI, Red de Sociología Jurídica en América Latina y el Caribe, 2012. P. 81 destaca que "la docencia hoy es facilitar el aprendizaje".
} 
(ii) Adquisición y consolidación de competencias.

Las competencias a adquirir durante el grado están planteadas de forma muy ambiciosa, quizás tanto, que a veces con el limitado tiempo que un cuatrimestre proporciona no se materializan de forma tan satisfactoria como gustaría, pues el Profesor en las reducidas horas de clase magistral debe explicar las instituciones y conceptos jurídicos básicos para que el alumno proceda posteriormente a resolver las cuestiones prácticas, sin tener lugar el planteamiento de debates sobre asuntos donde radica una divergencia en la doctrina o un problema en la aplicación y que generalmente incentivan la búsqueda de conocimiento y descubren la trascendencia de la rama del Derecho a tratar (más allá, por supuesto, de los siempre planteados debates sobre la interpretación romanista y germanista del artículo 464Cc o la ubicación del dolo como elemento subjetivo del tipo o en la culpabilidad, entre algunos otros).

Ante estas limitaciones, Fundamento Jurídico puede constituir una herramienta al servicio de la formación de los alumnos (con el mencionado apoyo de los Profesores) por los siguientes motivos:

- Constituye un instrumento para que los estudiantes se incentiven recíprocamente a continuar con su formación jurídica, ya que se plantean problemas conjuntamente, tratan de resolverlos y presentan a sus compañeros (muchas veces amigos) puntos de vista distintos para resolverlos. De hecho FONT RIBAS señala como problema en el trabajo en grupo que "en la mayoría de los casos no existe una discusión o una reflexión en el grupo de estudiantes, sino que simplemente se acepta la información tal y como proviene de cada estudiante encargado de recabarla" ${ }^{40}$. La dinámica establecida en el seno de Fundamento Jurídico de diálogo sobre cuestiones jurídicas que inquietan a los alumnos puede también imitarse en los trabajos en grupo, nueva competencia a desarrollar en los programas de grado.

- Complementa la labor docente desarrollada en las aulas por los Profesores porque, al no existir materias o asuntos tasados, los alumnos inicialmente escogen temas de actualidad que les interesan. Así por ejemplo, en el primer acto de Fundamento Jurídico (y en el primer número de su revista) se analiza la respuesta que el TEDH dio a la doctrina Parot o cuál fue la respuesta del Tribunal Supremo de Canadá a la conformidad con el Derecho Internacional de una posible independencia unilateral de Quebec.

- Facilita la relación profesor-alumno fuera de un sistema de evaluación. Como ha recordado uno de nosotros, "esto permite que haya una conjunción de intereses mayor: no se exige al alumno para que supere una prueba puesta por quien exige, sino que es otro quien examina"41 (en este caso el lector del respectivo artículo y su autoexigente redactor).

${ }^{40}$ FONT RIBAS, A, LLUÍS CABALLOL, A, GUAL DALMAU, M, GUASCH MARTORELL, R, GÓMEZ TRINIDAD, S, MORALES BARCELÓ, J, PONT VILADOMIU, C, ENZLER FANDÓS, A, "Las dificultades del aprendizaje autónomo en un contexto de ABP", Revista de Educación y Derecho, nº, 2009. Pág. 8.

${ }^{41}$ VIGIL DE QUIÑONES OTERO, D, "El estudio del Derecho en las oposiciones: posibles aportaciones a la vida universitaria", Docencia y Derecho, nº7, 2013. Pág. 4. 
- Introduce al alumno en lo que serían los primeros pasos de la labor investigadora, siendo en España una de los tres grandes bloques de salidas profesionales que ofrece el grado en Derecho (junto con el ejercicio de la profesión -también consultoría- y el estudio de oposiciones). Además, en el proceso de elaboración y maquetación de la revista se puede involucrar cualquier asociado aunque siempre haya un encargado principal, algo que permite un mejor manejo de las cada vez más usadas (y valoradas) TIC $^{42}$.

La profundización del alumno en los métodos de estudio nos parece una cuestión especialmente relevante que Fundamento Jurídico aporta y, por tanto, le dedicaremos un lugar autónomo en otro apartado para abordarlo con mayor profundidad.

(iii) Contextualización del estudio: actividades extraacadémicas.

En tercer lugar, identificamos como elemento positivo aportado por Fundamento Jurídico la contextualización del estudio a través de la realización de actividades extraacadémicas y el contacto con las instituciones. Utilizamos el término contextualización porque el Derecho como instrumento que regula nuestra vida en sociedad, aunque aparece en todos los ámbitos, dispone de unas instituciones que se dedican a su estudio y aplicación a través de las funciones que les han sido asignadas y cuyos miembros son licenciados (ahora graduados) en Derecho.

El conocimiento de cómo funcionan estas instituciones, la visita a sus lugares de trabajo y el contacto con los actuales integrantes de las mismas permite al alumno escuchar y reflexionar sobre las ideas y experiencias que sus miembros les aportan, pues pueden ampliar las variables a tener en cuenta a la hora de tomar futuras decisiones tan relevantes como el máster a cursar, la oposición que preparar o la rama del ordenamiento sobre la que investigar. Además, Fundamento Jurídico también se propone mantener un compromiso con la Universidad y colaborar con sus autoridades.

Sin ánimo de exhaustividad podemos mencionar algunas de las actividades que se desarrollaron durante los primeros meses de vida de la asociación. En primer lugar, se colaboró con el Vicedecanato de Alumnos encabezado en aquel momento por Consuelo Martínez-Sicluna, con quien se organizaron las jornadas de salidas profesionales. Asimismo, a título de asistencia se tuvo presencia en diversas ponencias o congresos organizados en la propia Facultad o fuera de ella como en la Real Academia de Jurisprudencia o en la Casa de Galicia. En segundo lugar, fuera de la Universidad se organizaron visitas al Tribunal Supremo y al Consejo General del Poder Judicial.

Merece la pena dedicar una especial referencia al Colegio de Registradores pues hoy actúa como un importante apoyo para hacer posible la existencia de Fundamento Jurídico al aceptar convertirse en patrocinador.

\section{Experiencia y métodos jurídicos.}

Otro aspecto que habría que destacar de la experiencia, ha sido el de la profundización de los alumnos en los métodos de estudio. Al iniciarse en la investigación, al hacer ejercicios de escritura que se publican, se han visto obligados a

\footnotetext{
${ }^{42}$ Sobre la importancia de las TIC DELGADO, A.M, -OLIVER, R, "Enseñanza del Derecho y tecnologías de la información y la comunicación”, Universidad Oberta de Cayalunya, 2003.
} 
manejar más métodos, y ello ha propiciado un avance significativo en la capacidad de estudio del Derecho.

Al respecto, querríamos destacar lo que Fundamento Jurídico ha supuesto sobre todo en el uso de los métodos inductivo y deductivo, exegético y dogmático.

En cuanto a los primeros, podríamos partir de que, con carácter general, el Derecho se estudia en las primeras etapas por el método deductivo: el alumno no sabe nada todavía y primero estudia la teoría para después ubicarla en la práctica ${ }^{43}$. Más o menos a eso responde la organización de magistrales y seminarios propia del sistema surgido de Bolonia. Llegado cierto momento, que normalmente coincide con el comienzo del ejercicio profesional, se produce el proceso inverso: un jurista comienza a trabajar y se encuentra con la necesidad de saber, de la teoría estudiada, qué parte es la aplicable al caso concreto que le ocupa. Se pasa entonces del método deductivo al método inductivo.

"Ambos son imprescindibles en la ciencia jurídica" dijo DE CASTRO ${ }^{44}$, quien notaba que "aunque con el primer método" -el deductivo- "se utilizarán más las ideas y en el segundo" -inductivo- "los conceptos, ambos son imprescindibles en la ciencia jurídica. Las ideas dan los criterios prácticos, los signos de orientación y los motivos de decisión. Los conceptos ofrecen los medios de ordenar la realidad jurídica y de comprenderla discursivamente. Se utilizarán conceptos definidores de contenido, ordenadores de materias y sistemáticos para dar forma unitaria al conocimiento" 45 . Al respecto añadía VALLET ${ }^{46}$ que "es preciso seguir complementariamente ambas vías con perspectiva universal y concreta a la vez" ${ }^{\natural 7}$. Como dice Don Jerónimo GONZÁLEZ

\footnotetext{
${ }^{43}$ Cuánto antes se logre ubicar lo que se ha estudiado en la realidad, antes se logra ser buen jurista. A éste respecto, creo que es muy interesante, es algo que puede ayudar, fijarse en qué experiencias prácticas tuvieron nuestros maestros, o nuestros referentes. Es curioso, pero en las biografías de muchos grandes juristas, se refiere que ya durante la carrera ayudaban a alguien o hacían algún tipo de práctica. Por ejemplo, dice la biografía que de don Joaquín Garrigues escribió el Prof. Alberto Bercóvitz, que "ya durante la carrera, inicia sus actividades profesionales al lado de su padre que era Relator-Secretario de la Audiencia Territorial". Dice también que "un año antes de terminar la carrera -en 1920- publica su primera obra", que fue un trabajo premiado por el penalista Luis Jiménez de Asúa. Finalmente, recuerda que de don Felipe Clemente de Diego aprende Garrigues a conciliar los estudios teóricos con el ejercicio de la profesión. Vid. BERCÓVITZ RODRÍGUEZ-CANO, A. "Biobliografía del Profesor Joaquín Garrigues “. En Estudios jurídicos en Homenaje a Joaquín Garrigues. Tecnos, Madrid 1971. Pag. XVI.

44 "Para no incurrir en las faltas habituales, conviene tener presente que la ciencia del Derecho positivo ha de considerar estos elementos: 1. La idea de la Justicia es el criterio organizador y decisorio de toda la realidad jurídica; incluso en el último y mínimo detalle, la idea de Justicia será la que justifique o excluya y la que dirá el porqué de la decisión. 2. El método: la naturaleza de esa idea es la que determina el procedimiento para conocer toda la realidad que ella informa. Por ello, en primer lugar, estará determinado por el criterio de la adecuación justa y sólo en segundo lugar por el de la adecuación lógica. El poder organizador de la idea se muestra al utilizar: a) el método deductivo, infiriendo criterios de adecuación de los principios generales de justicia (Derecho natural) y de los de la política (idea de la vida a realizar en un pueblo y en un tiempo determinado), y b) el método inductivo, conociendo la realidad social (estructura social, política y económica real) y la realidad jurídica (eficacia real de las normas en la vida social), para que sirva de base para la actuación jurídica (realización práctica en la materia jurídica)" Derecho civil de España. Madrid, 1987. Pag 42.

${ }^{45}$ Derecho civil de España. Madrid, 1987. Pag 42.

${ }^{46}$ Quien ha observado que Santo Tomás utiliza la doble vía, siendo deductivo en el Tratado de las Leyes ( $\mathrm{I}^{\mathrm{a}}, \mathrm{II}^{\mathrm{o}}$, q. 94) e inductivo en el Tratado de la Justicia (II ${ }^{\mathrm{a}}$, II ${ }^{\mathrm{a}}$, q. 57). Cfr. VALLET DE GOYTISOLO, J.B. "Perfiles jurídicos del Derecho Natural en santo Tomás de Aquino". En Estudios jurídicos en Homenaje al Profesor Federico de Castro. Tecnos. Madrid 1976.

${ }^{47}$ VALLET DE GOYTISOLO, J.B. "El Profesor Federico de Castro y el Derecho Natural". Anuario de derecho civil, ISSN 0210-301X, Vol. 36, N 4, 1983, págs. 1685-1726
} 
"puede destilarse de la observación empírica una verdad a posteriori que sirva luego de base para un procedimiento deductivo" $"$.

Ambos métodos son, por tanto, necesarios, para poder dominar el Derecho. Ahora bien, dado que la búsqueda de la adecuación justa es de algún modo más fácil pues los criterios de justicia son menos y están más presentes en el corazón humano, la labor ardua es la de adecuación lógica, pues requiere ser capaz de ver la realidad social y jurídica y conectar ambas. Requiere ser capaz de ubicar en la realidad social la realidad jurídica que estudiamos. Ésta labor se lleva a cabo mucho mejor por el método inductivo. De ahí que podamos decir lo siguiente: cuánto antes se sea capaz de comenzar a estudiar por el método inductivo, antes se será capaz de dominar el Derecho. Éste paso la mayoría de las personas sólo lo dan al comenzar a trabajar en la realidad práctica. Sin embargo, conviene comenzar antes a ser inductivo en el estudio. Y la labor de afrontar casos reales mediante artículos doctrinales es una buena forma de hacerlo, que ha funcionado muy bien en Fundamento Jurídico.

Por lo que respecta a los métodos dogmático y exegético, hay que recordar que el primero, sería el de asentar dogmas claros sobre las instituciones y a partir de ellos estudiar la ley, tomando por tanto todas en su conjunto; y el segundo, el que aboga por estudiar Derecho a base de interpretar cada ley vigente. Como recuerda don Jerónimo GONZÁLEZ ${ }^{49}$, la distinción tiene un valor especial en la técnica española como consecuencia de las aportaciones de ARCÁRATE y SANCHEZ ROMÁN. Según el primero, el método exegético expone el contenido de las leyes, Códigos y demás disposiciones mediante la exégesis y comentarios de los textos; el dogmático, después de determinar a priori las distintas instituciones jurídicas, toma las legislaciones en lo relativo a cada una. Según SÁNCHEZ ROMÁN, el método exegético toma como punto esencial el texto de la ley a cuya explicación se consagra, con sujeción a las reglas de la crítica y a los rectos principios de la hermenéutica; en tanto el dogmático considera las leyes en su conjunto, las organiza y hace su exposición sistemática, desarrollando los principios que contienen.

Normalmente, el estudio deductivo comienza siendo además dogmático (aunque los dogmas se asienten poco). Luego, a medida que el alumno se haga deductivo, se hará más exegético, pero sin perder lo dogmático, sobre todo si atendemos a la necesidad de los conceptos para una buena asimilación inductiva. Pero está claro que hay una diferencia muy grande entre un alumno que todavía no se enfrenta a la ley con método exegético (aunque al tiempo sepa ser dogmático), y el que ya emplea estos métodos.

Los artículos de fundamento jurídico han sido tanto dogmáticos como exegéticos. La realización de los mismos ha permitido a los estudiantes profundizar en el uso de ambos métodos.

\footnotetext{
48 GONZÁLEZ MARTíNEZ, J. "Métodos jurídicos". En Estudios Jurídicos. Registradores de EspañaThomson Reuters. Cizur Menor 2011. Pag 173.

${ }^{49}$ GONZÁLEZ MARTÍNEZ, J. "Métodos jurídicos”. En Revista Crítica de Derecho Inmobiliario Agosto de 1930, pag. 589; recogido en sus Estudios Jurídicos. Registradores de España- Thomson Reuters. Cizur Menor 2011. Pag 155.
} 


\section{Conclusiones: un método a imitar.}

Después de estos tres cursos (2014/2015, 2015/2016 y 2016/2017), podemos hacer un primer balance de la experiencia Fundamento Jurídico ciertamente positivo pues, en resumen, entendemos que puede servir como herramienta para alcanzar los siguientes tangibles e intangibles:

PRIMERO. Fundamento Jurídico como grupo humano constituye un medio pero también un fin en sí mismo. La creación de una dinámica de trabajo ha hecho sentirse a los alumnos motivados por el conocimiento del Derecho y sus repercusiones en cuestiones cotidianas y de actualidad; ha fomentado su compromiso e involucración con la Facultad; y la asunción de las responsabilidades inherentes a la llevanza y gobernanza de una asociación ha contribuido a su proceso de madurez personal.

SEGUNDO. No debemos renunciar a la idea de una Universidad residencial, pero lo cierto es que se antoja imposible que todos los alumnos vivan en Colegios Mayores de la Universidad (no existen plazas suficientes ni parece lo más conveniente para quien ya reside en Madrid), por lo que la creación de pequeños grupos o clubes de formación, estilo y vocación podría ser una solución.

TERCERO. La experiencia asociativa contribuye a un mejor estudio del Derecho en cuánto rama del conocimiento tan extensa que se hace inabarcable, lo cual nos coloca en una posición limitada que es más fácil de abordar aunando los conocimientos y soluciones que diversas personas pueden acumular.

CUARTO. Fundamento Jurídico ha entendido que debe tomar en consideración su labor y aportación a la Universidad, para lo cual también ha mantenido un cuidado en la estética y en el trato como muestra de respeto a la institución y como práctica ante los diferentes mundos del Derecho de que participarán sus asociados en un futuro.

QUINTO. En el contexto del EEES, Fundamento Jurídico (i) ha fomentado una relación profesor-alumno que la masificación de las aulas y el condicionamiento de la evaluación no siempre permiten, dado que el Profesor no solo ha transmitido conocimiento sino que ha aconsejado y orientado con su saber, creando dudas e identificando algunos de los medios necesarios para su resolución, lo cual es una forma de materializar esa nueva docencia basada en el aprendizaje del alumno; (ii) la dinámica de trabajo y diálogo puede potenciar la adquisición o consolidación de las ambiciosas competencias propuestas en los programas de grado como el trabajo en grupo o una mejor utilización de las TIC; (iii) las actividades extraacadémicas contextualizan el periodo de formación y brindan al alumno con la opinión y experiencias de profesionales del Derecho, como elemento a tener en cuenta en la decisión de su orientación profesional; y (iv) el compromiso y participación en la vida de la Facultad supone la creación de un sentimiento de comunidad universitaria que consideramos como beneficioso para el continuo desarrollo de la misma.

SEXTO. Por último, en cuanto a los métodos jurídicos la elaboración de artículos jurídicos supone un avance en el estudio del Derecho, lo que ha permitido el uso del método inductivo antes de lo que habitualmente se suele realizar (en el comienzo de la vida profesional). Asimismo, los alumnos han combinado tanto el método exegético como dogmático, afrontando satisfactoriamente la consolidación de algunas de las competencias propuestas en el programa de grado.

Efectivamente, todavía existen retos como alcanzar una mayor difusión de las publicaciones o el establecimiento de acuerdos oficiales con los Departamentos para 
institucionalizar y reconocer la labor de los Profesores que generosamente dedican su tiempo a los alumnos.

Mientras se están escribiendo estas líneas, Fundamento Jurídico ha renovado su Junta para afrontar el curso académico 2017/2018 con la misma voluntad de trabajo que en los cursos anteriores por lo que la ilusión por el proyecto (su principal motor) sigue vigente. En este punto no podemos sino celebrar que una iniciativa de estas características haya tenido cabida en nuestra Facultad, animando a darle continuidad por los beneficios que puede traer tanto para los alumnos como para la Universidad.

\section{Bibliografía.}

ALASTUEY DOBÓN, C, ESCUCHURI AISA, E, y MAYO CALDERÓN, B, en "Formación en competencias a través del estudio de la jurisprudencia. La experiencia en el Derecho Penal", Docencia y Derecho, n³, 2011

ARISTÓTELES. Etica a Nicómaco. Grammata, Granada 2008.

BALTHASAR. H. von, Herrlichkeit. I. Schau der Gestalt, 1961, trad. española, Gloria, vol. 1. La percepción de la forma, Madrid, 1985

BERCÓVITZ RODRÍGUEZ-CANO, A. "Biobliografía del Profesor Joaquín Garrigues “. En Estudios jurídicos en Homenaje a Joaquín Garrigues. Tecnos, Madrid 1971

BOTO ÁLVAREZ, A, en "El potencial de las revistas jurídicas de estudiantes en el EEES", Docencia y Derecho, nº4, 2012

CUBERO TRUYO, A, "Dificultades para la innovación docente en las áreas jurídicas. La compatibilidad entre la adquisición de competencias profesionales específicas y el cumplimiento de los objetivos globales", Revista de Educación y Derecho, $n^{\circ} 0,2009$

DE CASTRO, F. Derecho civil de España. Madrid, 1987

DE DIEGO VILLAGRÁN, E. en Pretorianos, de donde vienen y a donde van los fontaneros de la Moncloa, Martinez Roca, Madrid 2004

DE RIBERA. "Ayudar a engendrar: la figura del maestro en la Paideia Griega" en GRANADOS GARCÍA, J. y GRANADOS GARCÍA, J.A. La alianza educativa. Introducción al arte de vivir. Colección Didaskalos. Ed. Monte Carmelo. Burgos 2009

DELGADO, A.M, -OLIVER, R, "Enseñanza del Derecho y tecnologías de la información y la comunicación”, Universidad Oberta de Cayalunya, 2003

DOMINGO OSLÉ, R. Auctoritas. Ariel, 1999

D’ORS, A. Derecho privado romano, 8. Pamplona 1997

FONT RIBAS, A, LLUÍS CABALLOL, A, GUAL DALMAU, M, GUASCH MARTORELL, R, GÓMEZ TRINIDAD, S, MORALES BARCELÓ, J, PONT VILADOMIU, C, ENZLER FANDÓS, A, "Las dificultades del aprendizaje autónomo en un contexto de ABP", Revista de Educación y Derecho, n0, 2009

FRAGA IRIBARNE, M. Memoria breve de una vida pública. Planeta, Barcelona 1983 
GARCÍA DE ENTERRÍA contenido en FERNANDEZ- CARVAJAL, P. "Retrato de Eduardo joven". En Estudios sobre la Constitución española: homenaje al Profesor Eduardo García de Enterría. Civitas, 1991

GARRIGUES DÍAZ- CAÑABATE, J. "Don Felipe y la Universidad". Revista de Derecho Privado, t. XXIX, 1945

GARROTE DE MARCOS, M, "El TFG: sus modalidades, objetivos y competencias a validar. Reflexiones a partir de la experiencia de la Facultad de Derecho de la UCM", Docencia y Derecho, n9, 2015

GOMA LANZON, J. "Mayoría selecta" en El Pais, 12 de Enero de 2013

GONZÁLEZ MARTÍNEZ, J. "Métodos jurídicos". En Revista Crítica de Derecho Inmobiliario Agosto de 1930, pag. 589; recogido en sus Estudios Jurídicos. Registradores de España- Thomson Reuters. Cizur Menor 2011

GRANADOS GARCÍA, C. y GRANADOS GARCÍA, J. El Corazón: urdimbre y trama. Colección Didaskalos. Ed. Monte Carmelo. Burgos 2010

IBARRA SERRANO, F.J, "La formación jurídica en el S.XXI", en IBARRA SERRANO, F.J, ROJAS CASTRO, M.O, PINADO SOLORIO, M.E, La educación jurídica. Retos para el siglo XXI, Red de Sociología Jurídica en América Latina y el Caribe, 2012

LEWIS, C.S Los cuatro amores. $12^{\circ}$ ed. Rialp. Madrid 2008

LÓPEZ QUINTÁS, A. Estética de la creatividad. Juego. Arte. Literatura. Cátedra, Madrid 1977

ORTEGA DIAZ-AMBRONA, J.A Memorial de transiciones, Galaxia Gutemberg, Madrid 2015.

PLATON. Diálogos. Biblioteca clásica Gredos, Madrid 1987.

PAREJO GUZMÁN, M.J, "Innovaciones docentes mediante competencias en las ciencias jurídicas en el espacio europeo de educación superior", Docencia y Derecho, $\mathrm{n}^{\circ} 2,2010$

PEÑA BERNALDO DE QUIRÓS, M "En memoria de Don Federico de Castro y Bravo, nuestro Director”. Anuario de Derecho Civil. XXXVI-II. 1983

PROSSER y TRIGWELL (2006), GONZÁLEZ, C, MONTENEGRO, H, LÓPEZ, I.M y COLLAO, P, "Relación entre la experiencia de aprendizaje de estudiantes universitarios y la docencia de sus profesores", Calidad en la Educación, $\mathrm{n}^{\circ} 35,2011$

RIESCO GONZÁLEZ, M, "El enfoque por competencias en el EEES y sus implicaciones en la enseñanza y el aprendizaje", Tendencias Pedagógicas 13, 2008

ROCA, M.J. "La Forma estética personal y la cultura humanística universitaria”. Foro Nueva Época 4/2006

TURULL RUBINAT, M, ALBERTÍ ROVIRA, E, "El 'proyecto educativo de centro' en el ámbito universitario. Reflexiones y propuestas a partir de la experiencia de la Facultad de Derecho de la Universidad de Barcelona (2005-2016), Revista de Educación y Derecho, $\mathrm{n}^{\circ} 15,2016$

VALLET DE GOYTISOLO, J.B. "Perfiles jurídicos del Derecho Natural en santo Tomás de Aquino". En Estudios jurídicos en Homenaje al Profesor Federico de Castro. Tecnos. Madrid

VIDAL PRADO, C, "El espacio europeo de educación superior y su implantación en las universidades españolas", en Revista catalana de Dret Public, $\mathrm{n}^{\circ}$ 44, 2012 
VIGIL DE QUIÑONES OTERO, D. Consejos para opositar con éxito. CEU Ediciones, Madrid 2011

- "El estudio del Derecho en las oposiciones: posibles aportaciones a la vida universitaria", Docencia y Derecho, nº, 2013

- Breves consejos para estudiar Derecho con éxito. $2^{\mathrm{a}}$ Ed. Visión Libros. Madrid 2014 